\title{
Comparison Analysis of Resonant Controllers for Current Regulation of Selective Active Power Filter with Mixed Current Reference
}

\author{
Hao Yi ${ }^{*}$, Fang Zhuo ${ }^{*}, \mathrm{Yu} \mathrm{Li}^{*}$, Yanjun Zhang ${ }^{*}$, and Wenda Zhan ${ }^{\dagger}$ \\ ${ }^{* \uparrow}$ School of Electrical Engineering, Xi'an Jiaotong University, Xi'an, China
}

\begin{abstract}
Instead of extracting every selected harmonic component, the current reference of selective active power filter (APF) can be also obtained by filtering out the fundamental component from distorted load current for computation efficiency. This type of mixed current reference contains kinds of harmonic components and easily involves noises. In this condition, selective harmonic compensation must be realized by the current controller. With regard that selectivity is the most significant feature of controller, this paper presents specific comparison analysis between two types of resonant controllers: proportional-resonant (PR) controller and vector-resonant (VR) controller. The comparison analysis covers the relations, performances, and stability of both controllers. Analysis results conclude that the poorer selectivity of the PR controller could be relatively improved, but limitations from system stability make the improvement hardly realized. By contrast, the VR controller exhibits excellent selectivity and is more suitable for selective APF with mixed current reference. Experimental results from laboratory prototype validate the reasonability of analysis. And the features of each resonant controller are concluded.
\end{abstract}

Key words: Resonant controller, Selective active power filter, Selectivity, Stability margin

\section{INTRODUCTION}

The three-phase active power filter (APF), with its strong ability in dealing with harmonic current, is considered the most attractive solution in field of power quality control. The widely used APF is normally shunt connected between grid source and nonlinear loads. It operates as harmonics current source, injecting desired compensating current to cancel the corresponding harmonics components in distorted load current. Selective APF is an APF control scheme that can realize selective harmonics compensation. It provides meaningful benefits in practical fields, such as reasonable arrangement of limited device capacity to compensate the most harmful harmonics and flexible avoidance of potential resonance that may happen with grid nets [1].

Normally, the selectivity of APF is realized by the harmonic reference generator, by which every selected harmonic component is separately extracted. This method ensures clean

Manuscript received Apr. 23, 2013; revised Jun. 27, 2013

Recommended for publication by Associate Editor Kyo-Beum Lee.

${ }^{\dagger}$ Corresponding Author: yhflyer@163.com

Tel: +86-153-5358-0738, Fax: +82-29-8266-5223

${ }^{*}$ School of Electrical Engineering, Xi'an Jiaotong University, China current reference, but it requires high computation cost because multiple transformations and filtering are needed for every individual harmonic [2]-[4]. Some computation-efficient harmonic reference generators, such as high-pass filters [5], [6], subtraction fundamental components from load currents [7], and generating source current reference by power balance control from DC-voltage regulation [8], [9], are also available. These alternate methods are easier to implement, but the reference they generate is always mixed with kinds of harmonics. Thus, the selectivity of APF must be realized by the current controller in this condition.

Current regulation plays an essential role in the control of selective APF with mixed current reference. It should take dual functions of current regulation and selectivity. The proportional-integral (PI) controller in synchronous rotation frame (SRF) is widely considered as the best choice to satisfy these two functions [10], [11]. However, its implementation demands multiple rotation transformations for each selected harmonic, and this number would be doubled when both positive- and negative-sequence components need to be compensated, which means the PI controller in SRF is not a computation-efficient choice. 
Resonant controller would be a good alternative, if it can satisfy the dual functions of regulation and selectivity. The resonant controller is implemented in stationary frame and could regulate both positive- and negative-sequence components at one time, with zero steady-state error [12]-[16]. These features, together with computation-efficient reference generator mentioned above, would make the selective APF system quite computation-saving.

For the first function of current regulation, the resonant controller is sufficient to fulfill the task because of the internal principle, as introduced in Fukuda and Yoda [17]. However, the second function of selectivity, which is also an essential feature when face the mixed current reference, has not been thoroughly researched as the most important property by now.

The proportional-resonant (PR) controller is an extensively researched resonant controller. However, the research on this controller is mainly focused on the good performance of current regulation, on the basis that the current reference is accurately generated. The selectivity of the PR controller is briefly mentioned because this controller selectively provides infinite gains for the resonant frequency component [18]-[21]. In this condition, the following design guideline is suggested: the proportional term of the PR controller should be set as large as possible to enhance the transient speed [22], [23]. However, for the non-resonant frequency components, which may exist in the current reference, few concerns are carried out. The vector-resonant (VR) controller introduced in Lascu et al. [5] has a different structure and also presents good regulation for resonant frequency. However, the property of the VR controller has not been thoroughly analyzed by now.

In this paper, with purpose of implementing for selective APF with mixed current reference, selectivity is regarded as the most significant feature of resonant controller. The paper first presents detailed comparisons of the two types of resonant controllers, namely, the PR and VR controllers. The comparisons cover their relations with the SRF PI controller, their performance in current control loop, and their stability analysis, through which the features of each resonant controller is concluded. The controller suitable for the selective APF with mixed current reference is then chosen.

This paper is organized as follows. In Section II, the model of selective APF is set up. Section III gives introduction on the two resonant controllers, including their relations with the SRF PI controller and their parameter effects. The performances of both controllers in the current control loop are compared in Section IV, and their stability is analyzed in Section V. All conclusions from the comparisons are validated through a laboratory prototype in Section VI. Finally, the features of each resonant controller are concluded in Section VII.

\section{Model of SELEctive APF}

The selective APF studied in this paper is a normal three-phase type, as Fig. 1 shows, which is composed by

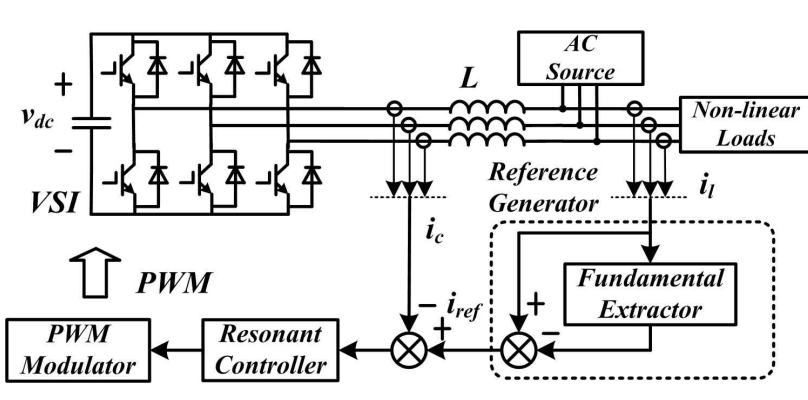

Fig. 1. Structure and basic control scheme of selective APF with mixed current reference, where $i_{l}$ is the load current; $i_{c}$ is the compensating current, with reference $i_{r e f} ; v_{d c}$ is the DC-link voltage.

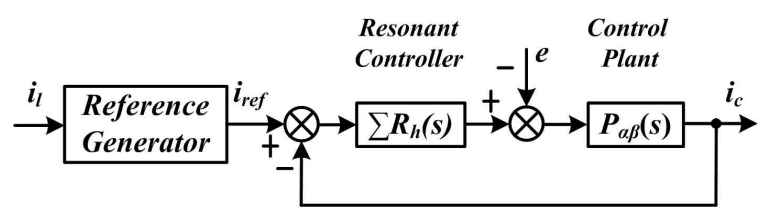

Fig. 2. Stationary frame model of selective APF with cascaded resonant controller $\left(\sum R_{h}(\mathrm{~s})\right.$, where subscript $h$ indicates selected harmonic orders).

three-phase voltage source inverter ( VSI), with one capacitor in the DC side and three line inductors in the AC side. The growing interest of replacing inductor by LCL filters is to achieve higher order attenuation for the switching frequency component. But it could also be modeled as an $L$ filter for simplification, because the concerned frequencies of selective APF are quite low compared with those of the switching frequency.

Selective harmonics compensation is normally realized by the current reference generator. However, this paper employs the reference generator in Fig. 1 for computation efficiency. The reference generator in Fig. 1 extracts fundamental component and subtracts it from the distorted load current. Since only fundamental component needs to be detected, the implementation is quite computation efficient; yet the price is that the current reference is mixed, pushing pressure of selective harmonics compensation to the current controller.

To satisfy the aforementioned demands and to further reduce computation burden, resonant controllers are employed because they are considered more computational efficient and because they feature zero-error regulation for AC signals. For the APF system, the resonant controllers are always implemented in a cascading form $\left(\sum R_{h}(s)\right.$ in Fig. 2), which is superposition of every individual resonant controller, with each one concerning about one selected harmonic. In this condition, the control scheme could be directly modeled (Fig. 2) in stationary frame, where $P_{\alpha \beta}(s)$ represents the system control plant and expressed as

$$
P_{\alpha \beta}(s)=\frac{1}{L(s+R / L)},
$$

where $L$ and $R$ are equivalent inductance and resistance of 
line inductor, respectively; the subscript $\alpha \beta$ indicates variable in stationary frame.

The gain effects of VSI are considered in the controller to simplify analysis, and related delay link is not shown in Fig. 2. Grid voltage $e$ is regarded as AC perturbation and could be eliminated by a properly tuned controller or by feed-forward compensation.

\section{INTRODUCTIONS OF RESONANT CONTROLLERS}

\section{A. Relation of SRF PI controller and Resonant Controller}

Different with the PI controller in SRF, the resonant controller is implemented in stationary frame, needless of rotation transformations. The relation of SRF PI controller and resonant controller will be discussed in this section, with simplified frequency domain rotation transformation between stationary frame and SRF which is deduced in the Appendix.

The SRF PI controller is set as (2), providing the zero point at $\omega_{z}$. It is believed to be sufficient in dealing with any signal, since the concerned signal has already turned to DC in its own SRF.

$$
P I_{d q}(s)=\frac{k\left(s+\omega_{z}\right)}{s}
$$

The PR controller is called "generalized integrator" in [14] and is considered able to regulate AC signal with zero error according to the internal principle. The relation of SRF PI controller and PR controller could be expressed as

$$
\begin{aligned}
P R(s) & =T_{P}^{-}\left[P I_{d q}(s)\right]+T_{N}^{-}\left[P I_{d q}(s)\right] \\
& =2 k \frac{s^{2}+\left(h \omega_{e}\right)^{2}+\omega_{z} s}{s^{2}+\left(h \omega_{e}\right)^{2}} \\
& =k_{P R}+\frac{s k_{P R} \omega_{z, P R}}{s^{2}+\left(h \omega_{e}\right)^{2}}
\end{aligned}
$$

where $T_{P}^{-}$and $T_{N}^{-}$are frequency domain inverse rotation transformations from SRF to stationary frame for positiveand negative-sequence components, respectively, with expressions shown in (A.8) and (A.10). $\omega_{e}$ is the angular frequency of grid voltage, indicating the fundamental frequency; $h \omega_{e}$ is the rotating frequency of SRF, implying corresponding harmonic frequency; $k_{P R}=2 k$ is proportional term of PR controller, and $\omega_{z, P R}=\omega_{z}$ is integral term.

Obviously, SRF PI controller and PR controller are actually equivalent, with difference that PI controller provides infinite gain for DC quantity in SRF, whereas the PR controller provides infinite gain for resonant frequency component in stationary frame. Nevertheless, both controllers do not consider the system control plant.

The system control plant in (1) could be transformed into SRF with (4) for positive sequence component and with (5) for negative sequence component.

$$
\begin{aligned}
& P_{d q, P}(s)=T_{P}^{+}\left[P_{\alpha \beta}(s)\right]=\frac{1}{L\left(s+R / L+j h \omega_{e}\right)} \\
& P_{d q, N}(s)=T_{N}^{+}\left[P_{\alpha \beta}(s)\right]=\frac{1}{L\left(s+R / L-j h \omega_{e}\right)}
\end{aligned}
$$

Here, $T_{P}^{+}$and $T_{N}^{+}$are frequency domain rotation transformations from stationary frame to SRF for positiveand negative-sequence components, with expressions shown as (A.6) and (A.9), respectively.

According to the definitions in (A.7), the imaginary term in (4) and (5) implies the cross-coupling relation of two axes in SRF ( $d$ and $q$ axes). Obviously, the real zero from SRF PI controller would never cancel the influences from complex pole of SRF system control plant. To decouple the influences, two methods could be considered: one is SRF-decoupled PI controller and the other one is SRF vector PI (VPI) controller [24], which is expressed as (6) for positive and (7) for negative.

$$
\begin{aligned}
& V P I_{d q, P}(s)=k \frac{s+R / L+j h \omega_{e}}{s} \\
& V P I_{d q, N}(s)=k \frac{s+R / L-j h \omega_{e}}{s}
\end{aligned}
$$

Obviously, The SRF VPI controller could provide corresponding complex zero to fully cancel the complex pole of SRF system control plant. Applying (A.8) and (A.10) as $(8)$, it is turned to stationary frame and called VR controller.

$$
\begin{aligned}
V R(s) & =T_{P}^{-}\left[V P I_{d q, P}(s)\right]+T_{N}^{-}\left[V P I_{d q, N}(s)\right] \\
& =k_{V R} \frac{s\left(s+\omega_{z, V R}\right)}{s^{2}+\left(h \omega_{e}\right)^{2}}
\end{aligned}
$$

where $k_{V R}=2 k$ is proportional term of VR controller, and $\omega_{z, V R}=R / L$ the integral term.

Therefore, the VR controller, which considers the system control plant, is equivalent to the SRF VPI controller.

Both PR controller and VR controller consider positiveand negative-sequence components, which mean resonant controller just needs only half of the number of the SRF PI (VPI) controller for selective APF control system, thus reducing the computation burden.

\section{B. Parameters Effects}

Every parameter effects are checked to assess their influences on the performances of resonant controllers.

\section{1) Parameters effects of PR controller}

The practical form of PR controller is damped PR (DPR) controller in (9) [12], where an additional damping factor $\omega_{c}$ makes it possible for digital implementation with limited precision.

$$
\operatorname{DPR}(s)=k_{P R}+\frac{s k_{P R} \omega_{z, P R}}{s^{2}+\omega_{c} s+\left(h \omega_{e}\right)^{2}}
$$




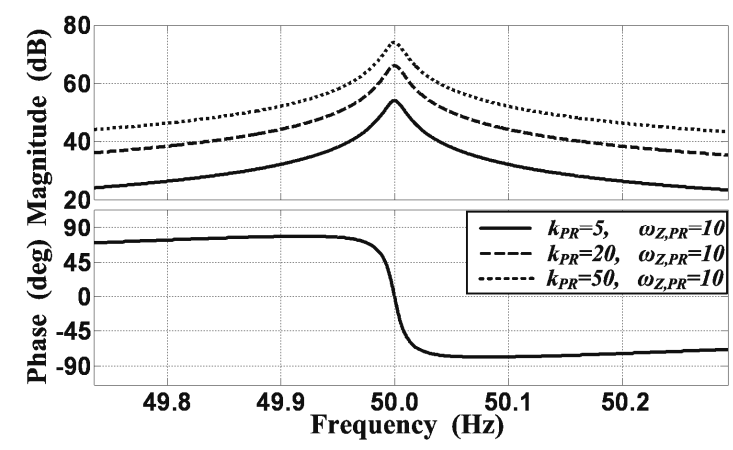

(a)

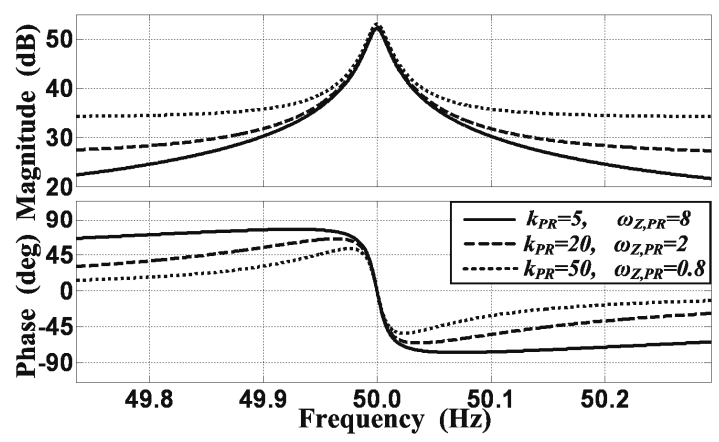

(c)

Fig. 3. Parameters effects of PR controller. (a) With different $k_{P R}$. different $\omega_{c}$.

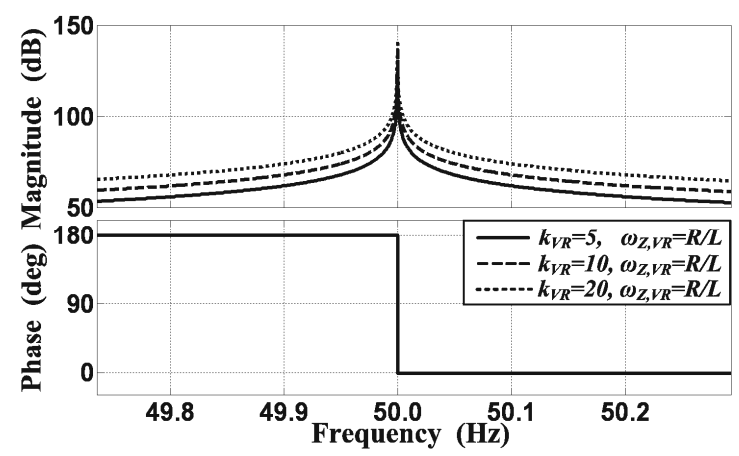

(a)

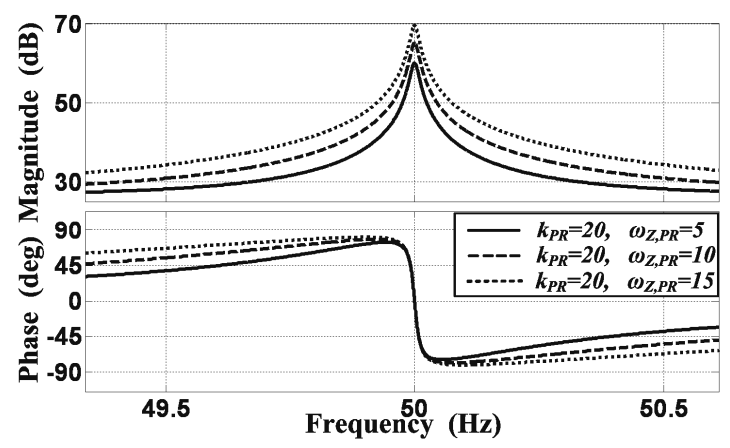

(b)

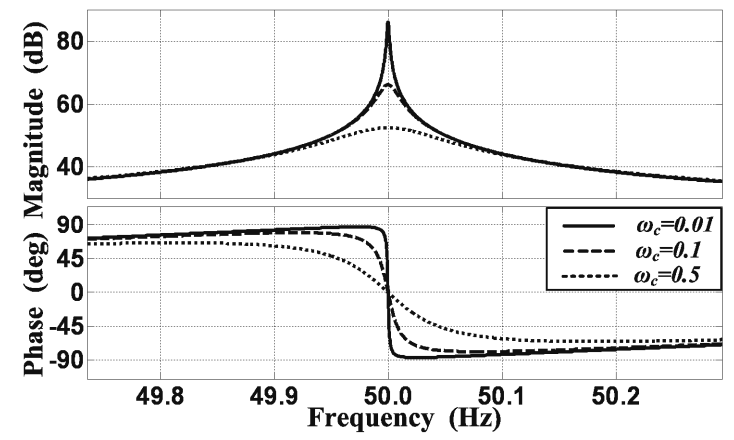

(d)

(b) With different $\omega_{z, P R}$. (c) with constant product of $k_{P R} \cdot \omega_{z, P R}$. (d) With

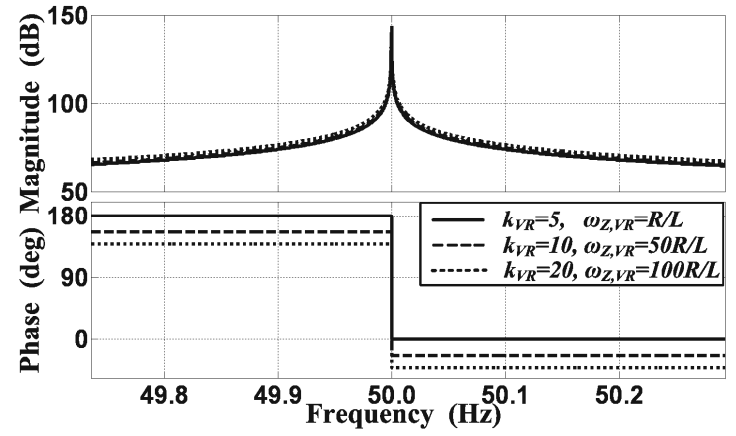

(b)

Fig. 4. Parameters effects of VR controller. (a) With different $k_{V R}$. (b) With different $\omega_{z, V R}$.

The PR controller has three parameters: proportional term $k_{P R}$, integral term $\omega_{z, P R}$, and damping factor $\omega_{c}$. Their effects are checked with different values in the bode diagrams of controller (see Fig. 3). The following properties could be observed:

a) With constant $\omega_{c}$, the peak of magnitude gain at resonant frequency provided by $\mathrm{PR}$ controller is determined by the product of $k_{P R} \bullet \omega_{z, P R}$, where $k_{P R}$ affects the magnitude gains for all frequencies, and $\omega_{z, P R}$ has more influences for frequencies around resonant one;

b) With constants $k_{P R}$ and $\omega_{z, P R}$, a larger $\omega_{c}$ has little influence to enlarge the effective bandwidth of controller, but it significantly decreases the magnitude gain around the resonant frequency. So it should be as small as possible, just meeting the basic precision demands of digital implementation.

\section{2) Parameters effects of VR controller}

The bode diagrams of VR controller is shown in Fig. 4, with two parameters checked. In Fig. 4(a), infinite gain appears at resonant frequency, and $k_{V R}$ governs the magnitude bandwidth of controller. The parameter $\omega_{z, V R}$ has great influence on system phase responses (see Fig. 4(b)) and should be tuned to cancel the SRF plant pole of the SRF system control plant.

\section{PERFORMANCES COMPARISON}

Cascaded resonant controllers are needed to control the selective APF. And the PR and VR controllers could be 


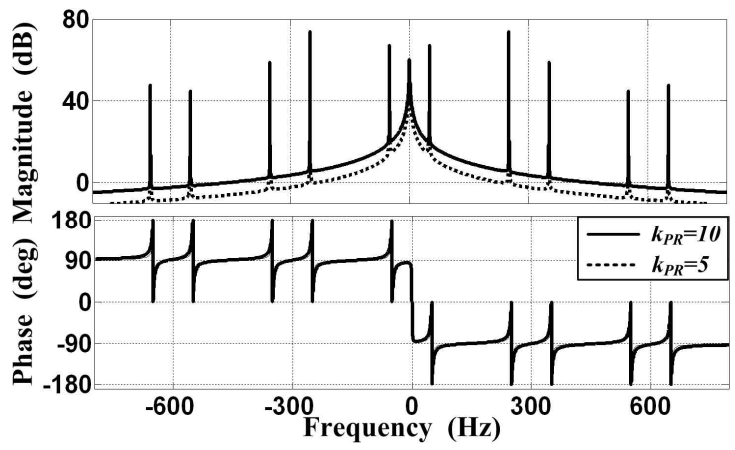

(a)

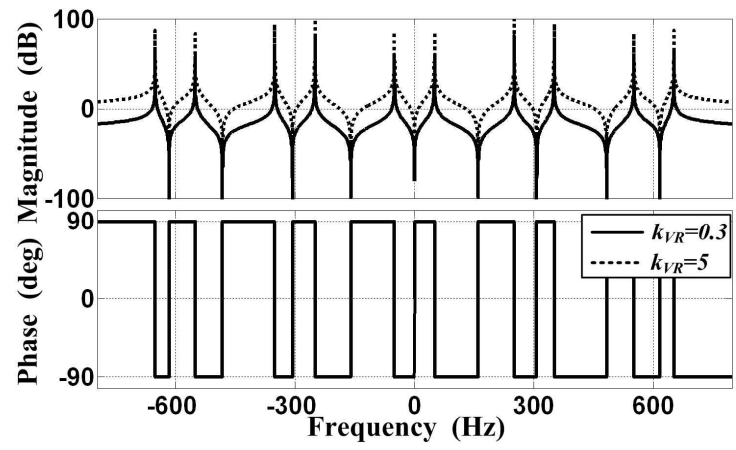

(b)

Fig. 5. Open-loop bode diagrams with cascaded resonant controllers. (a) With cascaded PR controllers. (b) With cascaded VR controllers.

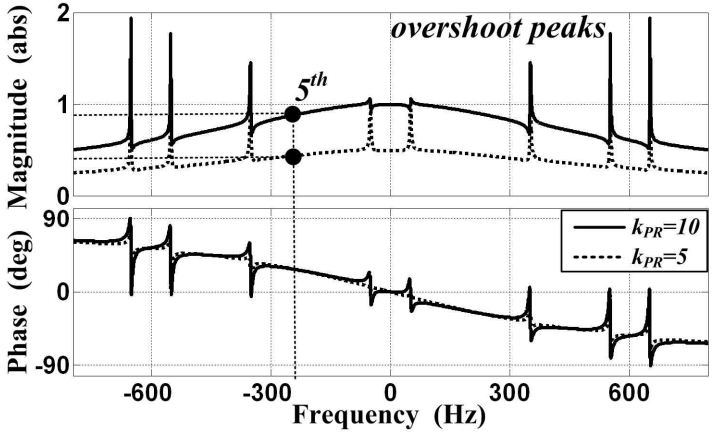

(a)

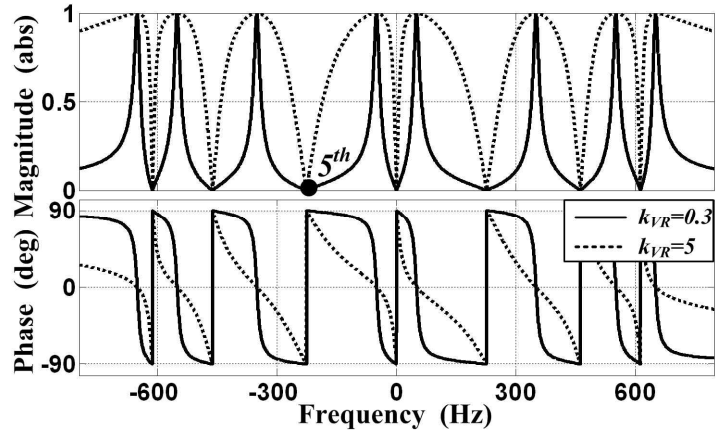

(b)

Fig. 6. Closed-loop bode diagrams with cascaded resonant controller. (a) With cascaded PR controller. (b) With cascaded VR controller.

TABLE I

SYSTEM PARAMETERS FOR COMPARISONS ANALYSIS

\begin{tabular}{|c|c|}
\hline Inductance of line inductor & $L=3.5 \mathrm{mH}$ \\
Resistance of line inductor & $R=0.01 \Omega$ \\
Fundamental frequency & $f_{1}=50 \mathrm{~Hz}$ \\
Selected harmonics orders & $h=1,5,7,11,13$ \\
\hline
\end{tabular}

expressed as $\sum P R$ and $\sum V R$, respectively, with each controller tuned on one selected harmonic. Both PR controller and VR controller have excellent current regulation performance for selected harmonics because large gain is assured for the resonant frequency (see Figs. 3 and 4). In this paper, the emphasis is put on their selectivity feature, with purpose of implementing for selective APF with mixed current reference. Selectivity is expected to be realized by flexible combination of cascaded resonant controllers, and the unselected components in the reference should not be output by removing the corresponding resonant controller from the cascaded controller.

The performance of both resonant controllers are compared with bode diagrams of the current control loop in Figs. 5 and 6 . And Table I lists the system values.

Fig. 5 illustrates the system open-loop bode diagrams, where (a) is with $\sum P R$ and (b) is with $\sum V R$; the negative frequency indicates negative sequence components. Both cascaded resonant controllers provide large magnitude gain around each resonant frequency, ensuring high reference tracking performance for the selected harmonics. However, for the non-resonant frequencies, the current control loop with $\sum P R$ shows low-pass features of system control plant (line inductor). And this feature is then amplified by its proportional term $k_{P R}$.

In conventional design guideline for $\mathrm{PR}$ controller, the current reference is supposed to be accurately generated and it is only formed by the selected harmonics. That means the non-resonant frequency components do not exist in the current reference. In this condition, $k_{P R}$ is suggested to be as large as possible to enhance system response speed.

However, for the selective APF with mixed current reference, the reference is mixed and easily involves noises. In this condition, larger $k_{P R}$ leads to larger gains for the unselected components (see Fig. 5(a)), causing degraded selectivity and weaker robustness. Furthermore, this influence is much more serious for low frequencies. And the worst situation happens at DC components, implying PR controller has no ability to reject DC noise that appears in the reference.

By contrast, the current control loop with $\sum V R$ presents good selectivity, with low gains for all the unselected components. $k_{V R}$ only affects the magnitude bandwidth, as shown in Fig. 5(b). 


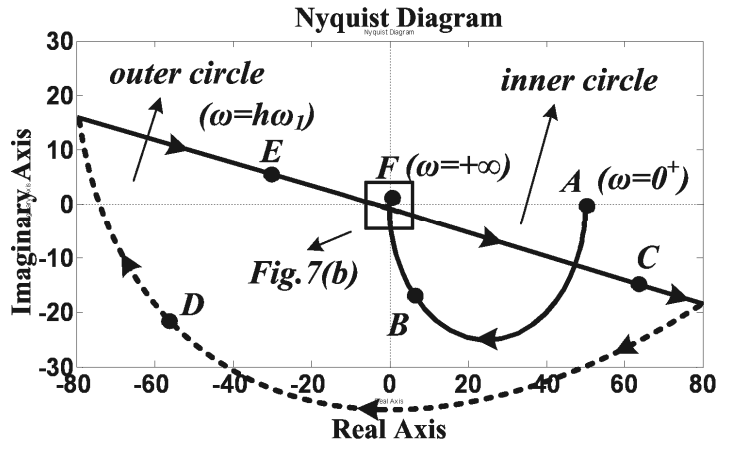

(a)

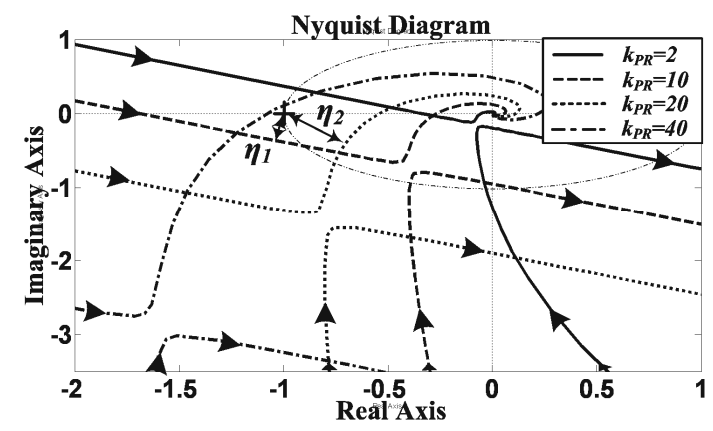

(b)

Fig. 7. Nyquist diagrams of current control loop with PR controller tuning on 11th-order harmonic. (a) For overall description. (b) For zoomed-in details on $(-1,0 j)$.

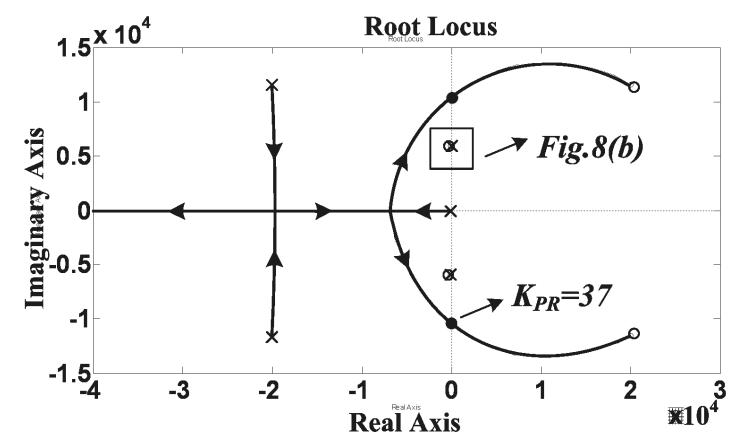

(a)

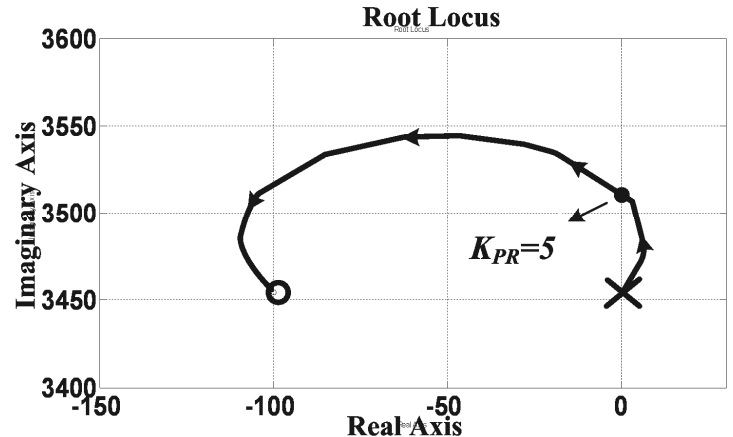

(b)

Fig. 8. Gain value selection through root locus diagrams of current control loop with PR controller tuning on 11th-order harmonic. (a) For overall description. (b) For zoomed-in details.

Similar conclusions could be achieved from the closed-loop bode diagrams in Figs. 6(a) and 6(b), in which the resonant controller that tuned on fifth-order harmonic is removed from both cascaded controllers to check their selectivity. Since reference generator in Fig. 1 is employed, every harmonics components, including the fifth-order, would still be included in the mixed current reference.

As shown in Fig. 6(a), certain fifth-order harmonic component in the reference would be output with $\sum P R$, even though the PR controller tuned on the fifth-order has been removed. That's because the controller has degraded back to the simple proportional controller at this frequency. According to Fig. 6(a), with $k_{P R}=10$, the ratio from outputting current to the reference at fifth-order harmonic is about $i_{c} i_{\text {ref }}=0.9$. It is unacceptable when resonance happens at the fifth-order harmonic in power distribution system. With similar reason, the noises in the mixed reference would be also output, according to $k_{P R}$.

On the other hand, the VR controller shows better selectivity, and $i_{c} / i_{r e f}=0$ for the fifth-order harmonic (Fig. 6(b)). The good selectivity of VR controller could be explained by its reference-tracking ability:

$$
\begin{aligned}
\frac{i_{c}(s)}{i_{r e f}(s)} & =\frac{P_{\alpha \beta}(s) \cdot \sum V R_{h}(s)}{1+P_{\alpha \beta}(s) \cdot \sum V R_{h}(s)} \\
& \approx \sum \frac{P_{\alpha \beta}(s) \cdot V R_{h}(s)}{1+P_{\alpha \beta}(s) \cdot V R_{h}(s)} \\
& =\sum \frac{s k_{b w}}{s^{2}+k_{b w} s+\left(h \omega_{e}\right)^{2}},
\end{aligned}
$$

where $k_{b w}=k_{V R} / L$.

The approximation is obtained according to the performance of VR controller (see Fig. 5(b)), where the VR controller provides almost zero gain for the non-resonant frequency, leading $i_{d} / i_{r e f}$ to zero, whereas providing nearly infinite gain around the resonant frequency, leading $i_{c} i_{\text {ref }}$ to unity. In this condition, the current control loop of selective APF performs as a multi-bands pass filter, with center frequency of each pass band selected by $\sum V R_{h}$, and the bandwidth adjusted by $k_{b w}$. This is identical with the closed-loop bode diagram in Fig. 6(b), in which performances with $k_{V R}=0.3$ and $k_{V R}=5$ are exhibited. Since the bandwidth is directly related to the response speed of the system, $k_{V R}$ should be as large as possible when the basic demand of selectivity is satisfied. 


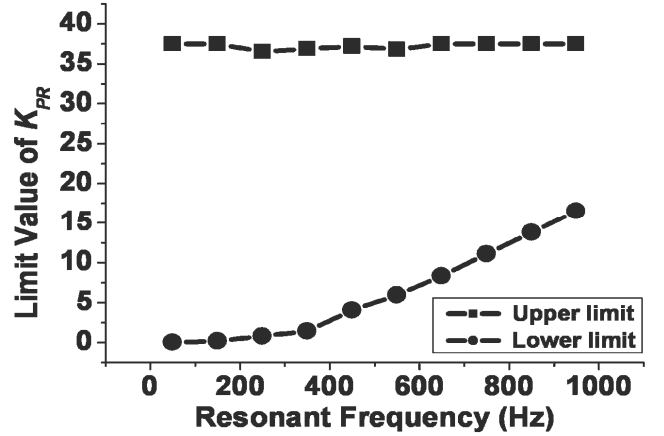

Fig. 9. Relation between value range of $k_{P R}$ and resonant frequency.

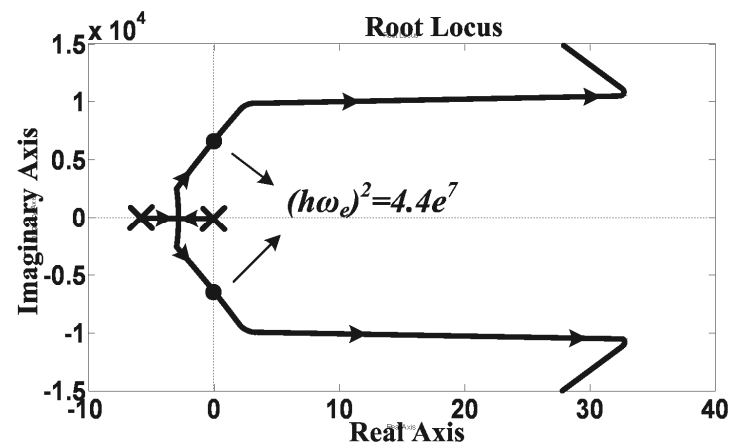

Fig. 10. Evaluation of highest order harmonic compensation through parameter root locus diagrams of current control loop with PR controller, in which $k_{P R}=34$.

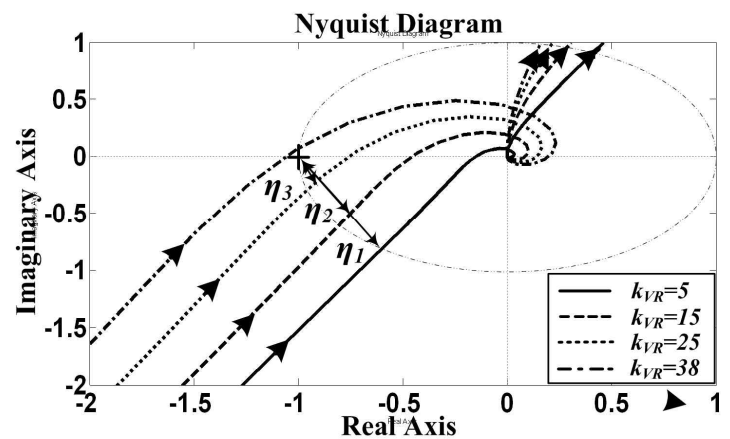

Fig. 11. Nyquist diagrams of current control loop with VR controller tuning on 11th-order harmonic (zoomed-in details on $(-1,0 j)$.

Moreover in Fig. 6(a), although unity gain is achieved for the resonant frequency in the current control loop with $\sum P R$, undesired overshoot peaks appear around each resonant frequency. These anomalous peaks indicate potential amplification for these inter-harmonic noises in the reference, resulting in low robustness to the mixed inaccurate reference. The amplifications may reach quite high level (see Fig. 6(a)) or may even lead system unstable. Whereas in Fig. 6(b), no overshoot peaks occur with $\sum V R$.

Unfortunately, when the reference generator in Fig. 1 is employed, this kind of noises is easily introduced into the reference, from measurement errors, inaccurate phase acquisition, or system transient change. The situation becomes worse when higher order harmonics need to be compensated, because limited sampling points will inevitably cause inaccurate reference for these high-frequency components.

From the aforementioned performance comparisons, it could be concluded that the PR controller is less selective, and its performances are quite sensitive to the noises in the reference; whereas the VR controller shows good selectivity and robustness.

According to Figs. 5 and 6, the proportional terms of both controllers show significant influences on their performances. The poorer selectivity of the PR controller could be relatively improved by reducing $k_{P R}$ (Figs. 3(a), 5(a), and 6(a)). However, it could never eliminate the influences from the unselected components, especially from the low-frequency components, because of its low pass feature. The influences and limitations of reducing $k_{P R}$ will be discussed in detail in the next section, from view of system stability.

\section{CONSIDERATION OF SYSTEM STABILITY}

With analysis above, it could be seen that the proportional term has significant impacts on performances of both resonant controllers, and its value selection should take system stability into consideration.

Digital delay should be considered in the system model (Fig. 2) when analyzing system stability. The delay is formed by the sampling time, digital calculation time, and zero-order hold effect of pulse-width-modulated (PWM) inverter. And it could be described with Padé approximation in (11), where $T_{d}$ is the delay time and equals to $3 / 2$ sampling time [7].

$$
e^{-T_{d} s} \approx \frac{1-T_{d} s / 2+\left(T_{d} s\right)^{2} / 12}{1+T_{d} s / 2+\left(T_{d} s\right)^{2} / 12}
$$

To achieve better selectivity, the current control loop with cascaded resonant controllers always have several $0 \mathrm{db}$ crossings in the open-loop bode diagram, as shown in Figs. 5(a) and 5(b). In this condition, conventional phase margin becomes unreliable to indicate system stability margin [25]. Therefore, vector margin introduced by Smith should be employed to measure the proximity of stability [16], [26]. Vector margin quantifies the stability margin as the nearest distance from the system open-loop transfer function to the critical point $(-1,0 j)$ in Nyquist diagram, giving exact conclusion on system stability. The vector margin could be expressed as (12), where $G(s)$ is the system open-loop transfer function.

$$
\eta=|1+G(s)|
$$

\section{A. System stability with PR controller}

Taking the PR controller with resonant frequency at 11th-order harmonic as example, the Nyquist diagrams of the 


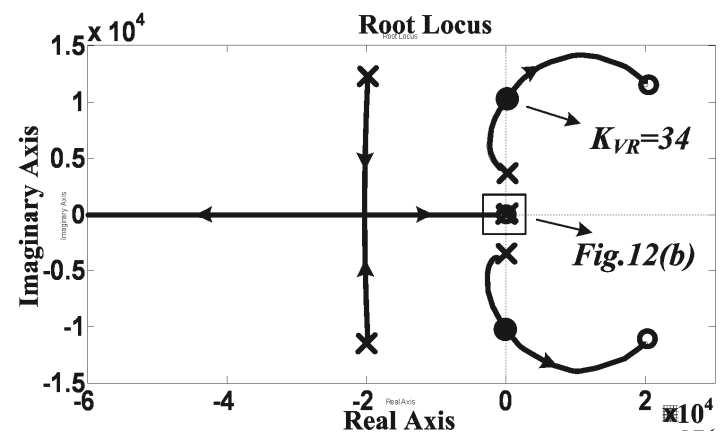

(a)

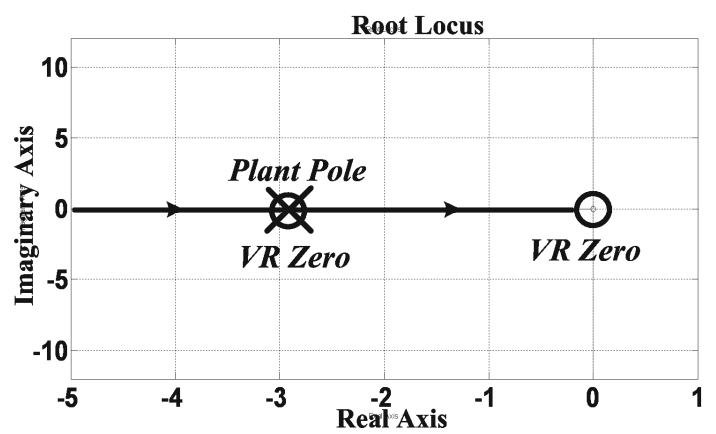

(b)

Fig. 12. Gain value selection through root locus diagrams of current control loop with VR controller tuning on 11th-order harmonic. (a) For the overall description. (b) For zoomed-in details of pole/zero cancellation.

current control loop are shown in Fig. 7, where (a) is for the overall description and (b) is for the zoomed in details on $(-1$, $0 j)$.

In Fig. 7(a), the Nyquist diagram is drawn with increasing of frequency from $\omega=0^{+}$to $+\infty$, along points A to F. And the negative sequence components with negative frequencies from $\omega=-\infty$ to $0^{-}$are symmetrical about real axis and are not shown in the figure. The area of the inner circle indicates magnitude gains for the non-resonant frequencies of scheme with PR controller, whereas the outer circle implies gains for the resonant frequency. For the selective APF with mixed current reference, the inner circle is expected to be smaller to improve the selectivity, while the outer circle needs to be as large as possible to guarantee tracking performance for the selected harmonics. According to the analysis in Section IV, the $k_{P R}$ of PR controller should be reduced to make the inner circle smaller to improve selectivity.

Zoomed-in details are illustrated in Fig. 7(b), in which several $k_{P R}$ are taken to check system stability. Note that the value of $\omega_{z, P R}$ should be correspondingly changed to ensure the constant high gains for the selected harmonic (Fig. 3(c)). It is worthy to note that larger and smaller $k_{P R}$ would all lead system unstable $\left(k_{P R}=40\right.$ or 2 in Fig. $\left.7(\mathrm{~b})\right)$, with which the critical point $(-1,0 j)$ is encircled in clockwise direction. Moreover, in stable conditions, the system vector margin $\left(\eta_{1}\right.$

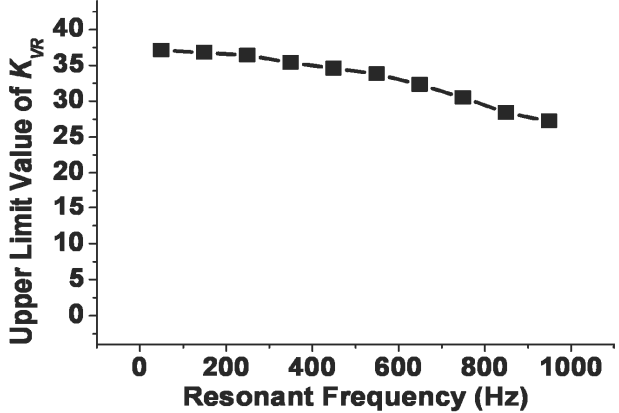

Fig. 13. Relation between value range of $k_{V R}$ and resonant frequency.

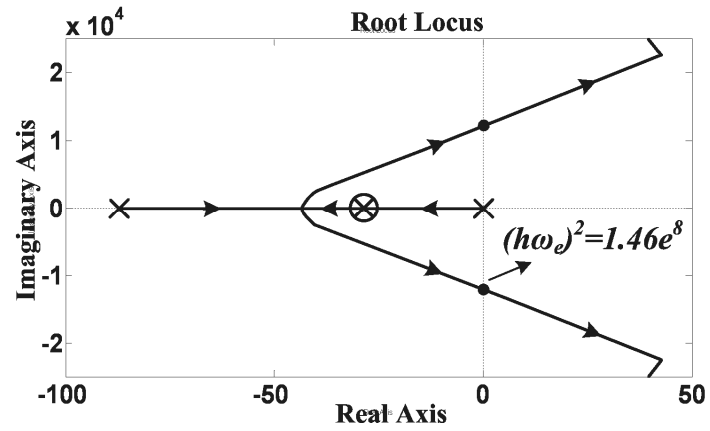

Fig. 14. Evaluation of highest order harmonic compensation through parameter root locus diagrams of current control loop with VR controller, in which $k_{V R}=0.3$.

and $\eta_{2}$ ) is also determined by $k_{P R}$, which means that $k_{P R}$ exhibits direct influences on system stability, and its value should be proper tuned with upper and lower limits. This conclusion is more reasonable than the conventional one with phase margin, which only focused on the upper limit of $k_{P R}$ [12], [22], [23].

Moreover, this conclusion is also helpful in assessing the value range of $k_{P R}$ and in evaluating the highest order harmonic that can be compensated by the selective APF.

When the resonant frequency of PR controller has been determined by the selected harmonic, the value range of $k_{P R}$ could be obtained by system root locus diagram in Fig. 8, from which the upper and lower limit values of $k_{P R}$ could be achieved, from view of stability. In Fig. 8, PR controller for the 11th-order harmonic is taken as example, and the value of $k_{P R}$ should be in the interval of $(5,37)$. A larger or lower value would all lead the locus going to the right half plane in the diagram.

Note that this interval is determined by the highest resonant frequency in cascaded PR controller, and it will be narrower when higher order harmonics are selected. The corresponding relation between value range of $k_{P R}$ and resonant frequency is depicted in Fig. 9, in which the lower limit would increase with the growth of resonant frequency. 


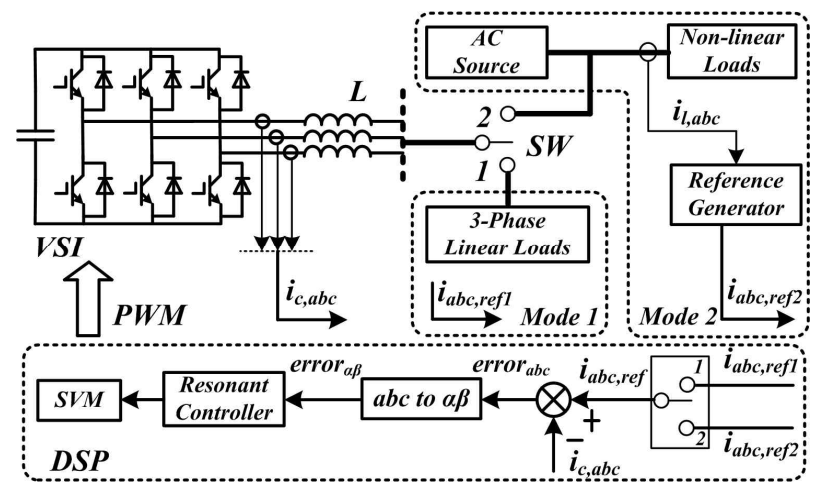

Fig. 15. Schematic diagram of laboratory prototype.

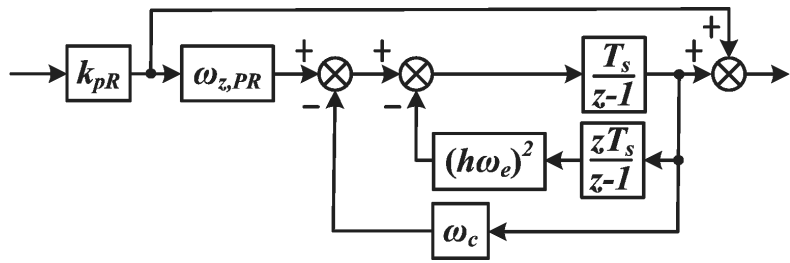

(a)

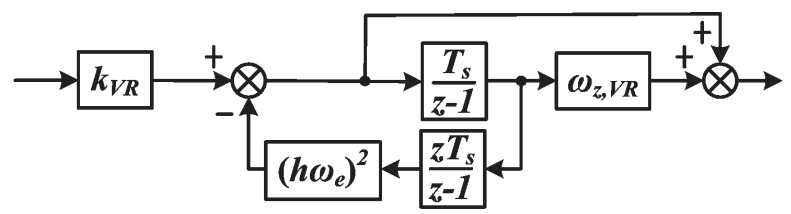

(b)

Fig. 16. Digital implementation of resonant controllers, based on dual interconnected integrators. (a) For PR controller. (b) For VR controller.

Therefore, the method, which improves the selectivity of PR controller by reducing $k_{P R}$, is just relatively effective. And this method would be hardly effective when higher order harmonics are selected.

Additionally, when $k_{P R}$ has already been selected, the highest order harmonic that can be compensated could be also evaluated. In Fig. 10, the parameter root locus diagram describes system poles mapping along with increasing of resonant frequency square $\left(h \omega_{e}\right)^{2}$. With $k_{P R}=34$, the limit of $\left(\mathrm{h} \omega_{\mathrm{e}}\right)^{2}$ for the PR scheme is almost $4.4 e^{7}$, which means that for typical nonlinear loads with harmonics current at orders of $6 k_{h} \pm 1\left(k_{h}=1,2,3 \ldots\right)$, the highest order harmonic that could be compensated by selective APF is 19th. These findings are identical with results reported in Bojoi et al. [7].

\section{B. System stability with VR controller}

Still taking resonant frequency at 11 th-order harmonic as example, the corresponding Nyquist diagram with VR controller is depicted in Fig. 11. Only one large circle for the resonant frequency is observed, and it is almost zero for the others, indicating good selectivity. Similarly, several proportional terms $k_{V R}$ are adopted to check their influences on stability. The figure shows that system stability is also
TABLE II

EXPERIMENTAL PROTOTYPE PARAMETERS

\begin{tabular}{|c|c|}
\hline Grid frequency (fundamental) & $f_{1}=50 \mathrm{~Hz}$ \\
Line inductor (inductance) & $L=3.5 \mathrm{mH}$ \\
DC voltage & $V_{D C}=180 \mathrm{~V}$ \\
Switching frequency & $f_{\mathrm{s}}=10 \mathrm{k} \mathrm{Hz}$ \\
Resistor loads (for mode 1) & $R_{1}=13 \Omega$ \\
AC line-line voltage (for mode 2) & $V_{A C}=90 \mathrm{~V}$ \\
Resistor loads (nonlinear loads for mode 2) & $R_{1}=3.5 \Omega$ \\
\hline
\end{tabular}

determined by the value of $k_{V R}$, and its value has only upper limit (the circle with $k_{V R}=38$ has encircled the critical point in clockwise direction).

The value range of $k_{V R}$ is obtained from the root locus diagrams of VR scheme in Fig. 12, in which the resonant frequency is 11th-order harmonic. Obviously, VR controller presents a real zero to cancel the influences of inductor plant pole (see Fig. 12(b)). And its proportional term has only upper limit: $k_{V R}<34$ (see Fig. 12(a)). The relation between upper limit of $k_{V R}$ and the resonant frequency is depicted in Fig. 13, in which the value limit is slightly decreased with growth of resonant frequency. Therefore, selection of $k_{V R}$ is much easier and is flexible tradeoff between the selectivity, stability, and response speed of the system.

Evaluation of the highest order harmonic that can be compensated is obtained with the parameter root locus diagram in Fig. 14, where $k_{V R}$ has already been determined as 0.3 for good selectivity. In this condition, the highest order that could be compensated is 35th for the typical nonlinear loads (the limit value of resonant frequency square $\left(\mathrm{h} \omega_{\mathrm{e}}\right)^{2}$ is almost $1.46 \mathrm{e}^{8}$ ). Note that this limit value will slightly decrease with increasing of $k_{V R}$. But the limit value is still larger than the scheme with PR controller, indicating VR controller could compensate higher order harmonic components.

\section{EXPERIMENTAL VALIDATION}

\section{A. Prototype introduction}

\section{1) Prototype setup}

To validate the analysis in this paper, a $10 \mathrm{kVA}$ laboratory prototype is established, with schematic setup shown in Fig. 15, and the parameters listed in Table II.

Experiments are carried out in two modes (see Fig. 15): in mode 1 , switch $S W$ is thrown to point 1 , and $V S I$ is directly connected to the loads, working as harmonic currents generator; in mode $2, S W$ is thrown to point 2 , and VSI is shunt connected between source and nonlinear loads, working as selective APF. Current control loops in both modes have similar reference tracking task. However, the current references of the modes are different. The reference in mode 1 is given by codes, whereas the reference in mode 2 is obtained by the reference generator in Fig. 1. The reason of 


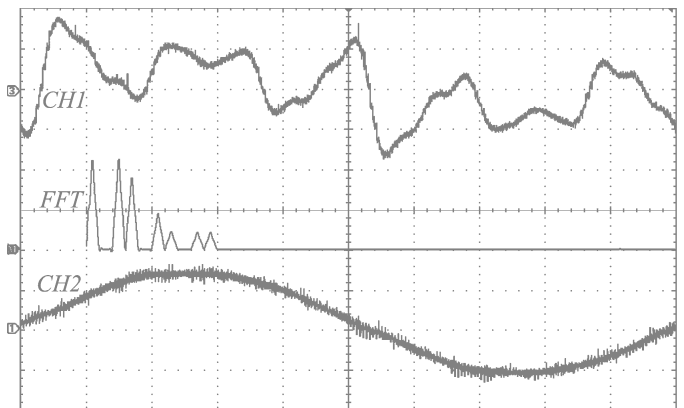

(a)

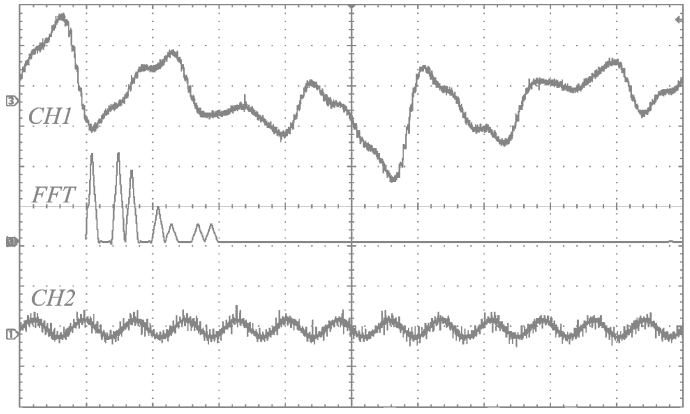

(c)

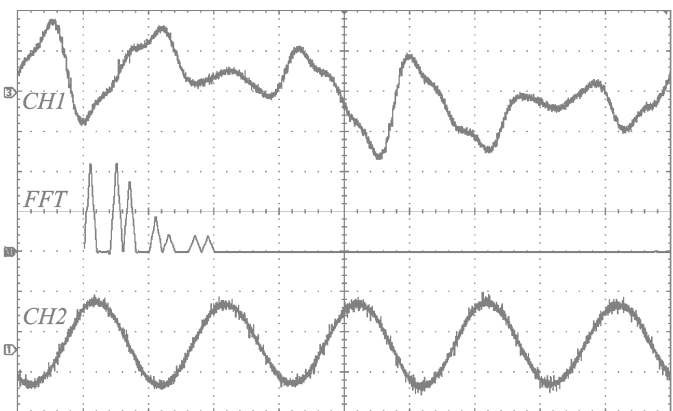

(b)

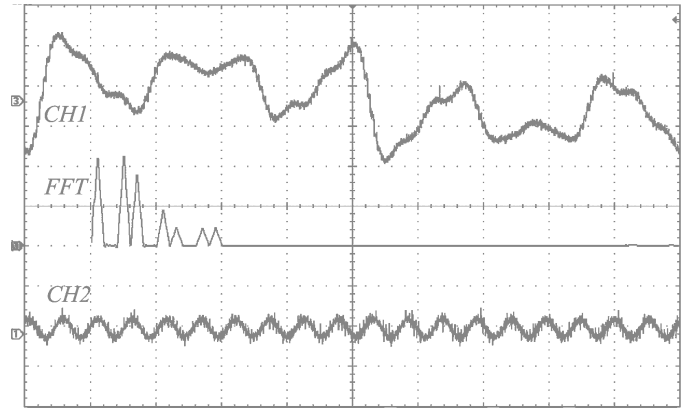

(d)

Fig. 17. Selectivity check for VR controller in mode 1, with time scale: $2 \mathrm{~ms} / \mathrm{div}$, where $\mathrm{CH} 1$ is the mixed current reference from DA, scale: $1 \mathrm{~V} /$ div; FFT represents the fast Fourier transform analysis of reference, scale: $500 \mathrm{~Hz} / \mathrm{div}$; $\mathrm{CH} 2$ is the outputting current, scale: 5 A/div. (a) Fundamental component selected. (b) 5th-order component selected. (c) 13th-order component selected. (d) 19th-order component selected.

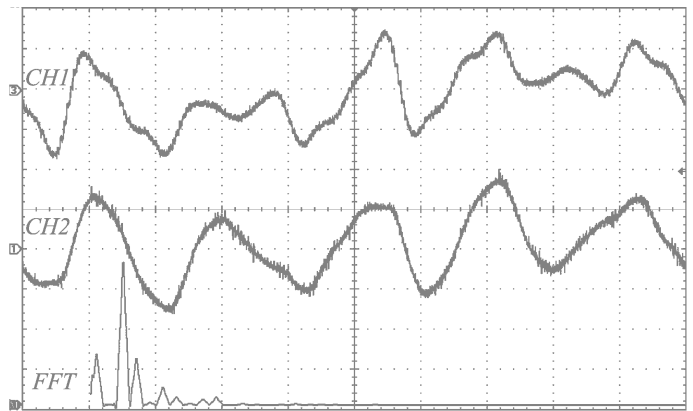

(a)

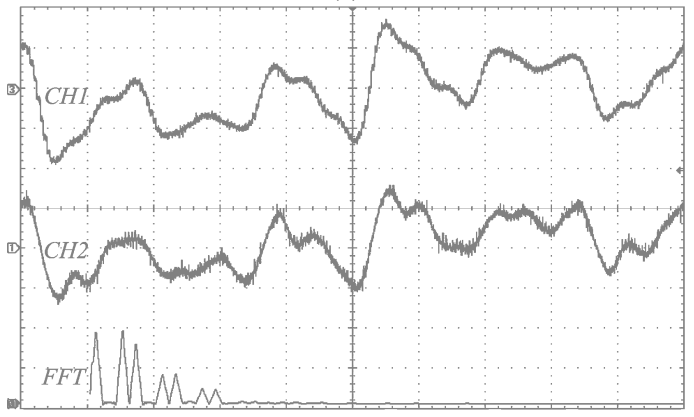

(c)

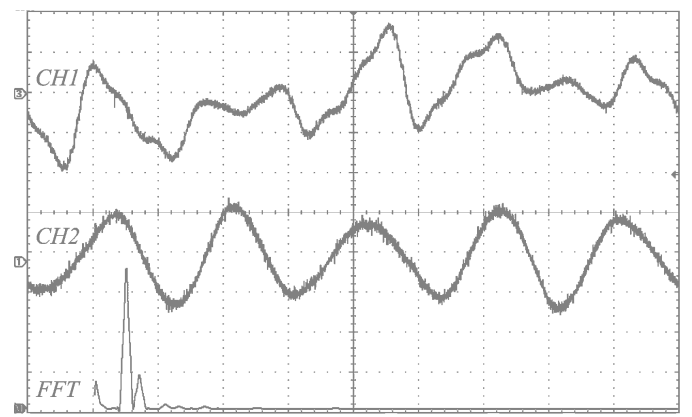

(b)

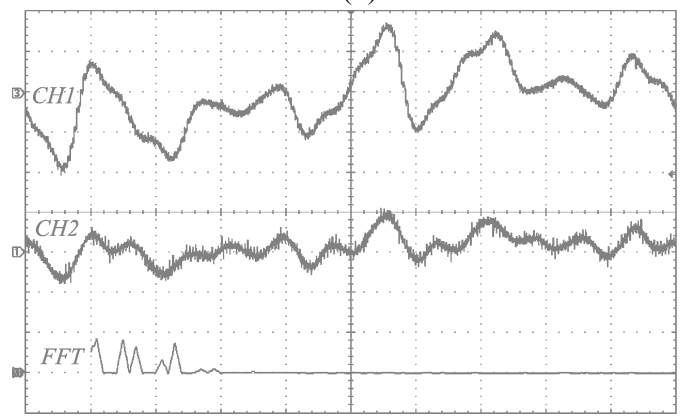

(d)

Fig. 18. Selectivity check for PR controller in mode 1, with time scale: $2 \mathrm{~ms} / \mathrm{div}$, where $\mathrm{CH} 1$ is mixed current reference with same content in Fig.17, scale: $1 \mathrm{~V} /$ div; $\mathrm{CH} 2$ is the outputting current, scale: $5 \mathrm{~A} /$ div; FFT is the fast Fourier transform analysis of the outputting current, scale: $500 \mathrm{~Hz} / \mathrm{div}$; (a) 5 th-order component selected and $k_{P R}=10$. (b) 5 th-order component selected and $k_{P R}=0.5$. (c) 13th-order component selected and $k_{P R}=20$. (d) 13th-order component selected and $k_{P R}=6$. 


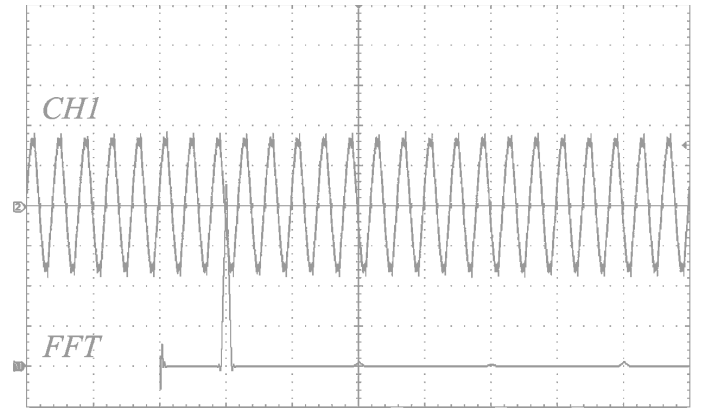

(a)

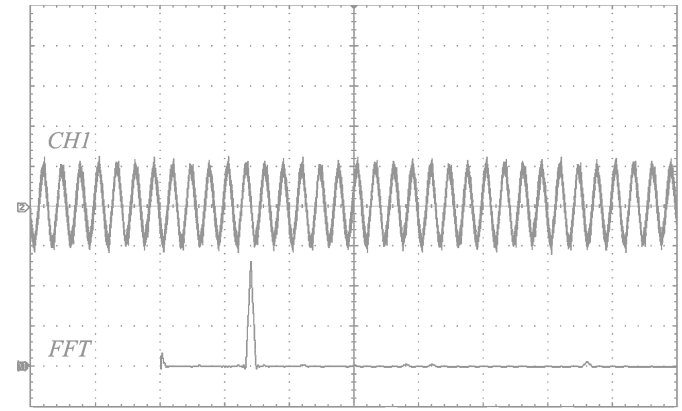

(b)

Fig. 19. Test of highest order harmonic outputting with VR controller in mode 1, with time scale: $2 \mathrm{~ms} / \mathrm{div}$. where CH1 is outputting current, scale: 2 A/div; FFT with scale: $1.25 \mathrm{kHz} /$ div. (a) Outputs 25 th-order harmonic current. (b) Outputs 35 th-order harmonic current.

\section{2) Control scheme realization}

All the necessary control schemes, including signal measurement, reference generation, current control, PWM modulation, and related logic control, are implemented with single digital signal processor (DSP TI: TMS320F2812).

The PR and VR controllers are implemented in stationary frame, with dual interconnected integrators as depicted in Figs. 16(a) and 16(b), respectively. Both controllers are discretized in the same way: the direct integrator is discretized in forward Euler method and the feedback integrator in backward Euler method, which is the most accurate discretization method to keep positions of resonant poles [27].

\section{B. Experiment results}

\section{1) Mode 1}

In this mode, the three-phase VSI is working as a harmonic current generator, with reference given by codes. Mixed current reference is employed to check the selectivity of the PR and VR controllers. The mixed reference contains several harmonics with orders of $1 \mathrm{st}, 5 \mathrm{th}, 7 \mathrm{th}, 11 \mathrm{th}, 13 \mathrm{th}, 17 \mathrm{th}$, and 19 th, and the proportion of each order is illustrated by FFT analysis in Fig. 17. Only one order component is selected by the corresponding resonant controller, and the output currents are shown in Fig. 17 for the VR controller and Fig. 18 for the PR controller.

The VR controller exhibits excellent selectivity, as shown in Fig. 17, in which every single selected component is output with its corresponding proportion in the reference, and the other unselected components in the mixed reference are entirely filtered out. In the experiment, $k_{V R}$ is selected as 0.5 and $\omega_{z, V R}$ is tuned according to the line inductor and resistor load for each order component. Good selectivity and tracking performances are achieved for components up to the 19th order.

By contrast, with the same mixed current reference, the selectivity of the PR controller is poorer. In the test, $k_{P R}$ could be selected as large as 30 to keep the system properly working. However, as shown in Fig. 18(a), where the fifth-order is selected, many unselected components are still output even though the smaller $k_{P R}\left(k_{P R}=10\right)$ is chosen. The contents of the output current are depicted by FFT analysis, in which the first- and seventh-order components are still beyond $1 \mathrm{~A}$ (RMS value). When $k_{P R}$ is reduced to 0.5 , all unselected components could be suppressed below 1 A (see Fig. 18(b)), with selected fifth-order component still perfectly tracked. However, it is still unacceptable for some crucial condition. The further reduction of $k_{P R}$ has almost no effect in improving the selectivity, indicating that the PR controller could not totally eliminate the influences from unselected components because of its low-pass feature.

When 13th-order component is selected by the PR controller, the output current and the corresponding FFT analysis are shown in Figs. 18(c) and 18(d), with $k_{P R}$ adopted as 20 and 6 , respectively. The relation between $k_{P R}$ and selectivity could be also observed, and the further reduction of $k_{P R}$ would lead the system unstable in the test. Therefore, $k_{P R}=6$ almost reaches the lower limit of system stability, confirming the conclusion from Section V: $k_{P R}$ has upper and lower limits from view of system stability and its lower limit will increase with the growth of the resonant frequency.

The test in Fig. 18 validates the conclusion that the selectivity of PR controller could be relatively improved by reducing $k_{P R}$, but it has limitation from system stability, particularly when higher order harmonic is selected.

The highest order harmonic regulation is also tested in the experiment. With $k_{P R}=20$, the highest order harmonic that could be output with PR controller is 19th order, in which condition there is almost no possibility to improve system selectivity because of system stability. In contrast with VR controller, good selectivity and tracking performance could be achieved for the 19th-order harmonic, as depicted in Fig. 17(d). Higher order harmonic could be also output, as shown in Fig. 19, where (a) indicates the 25th-order harmonic output by the VR controller and (b) for the 35 th order. 


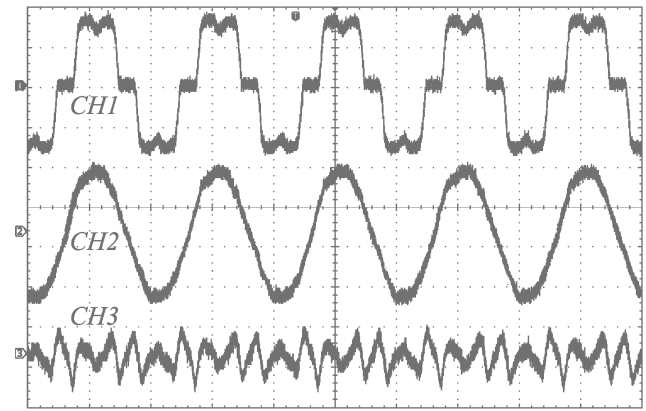

(a)

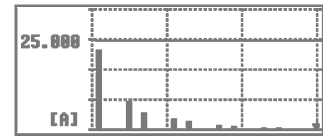

Spectrum 1

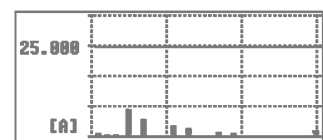

Spectrum 3

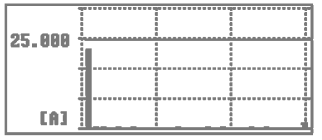

Spectrum 2

(b)

Fig. 20. Harmonics filtering performances with VR controller in mode 2, (the PR controller has similar performances and not shown separately). (a) Waveforms with scales of $20 \mathrm{~A} / \mathrm{div}$ and $10 \mathrm{~ms} / \mathrm{div}$ for three channels. CH1 is the load current; CH2 is the source current; $\mathrm{CH} 3$ is the compensating current. (b) Corresponding spectrums with scale $500 \mathrm{~Hz} /$ div. Spectrum 1 represents the load current. Spectrum 2 represents the source current. Spectrum 3 represents the compensating current.

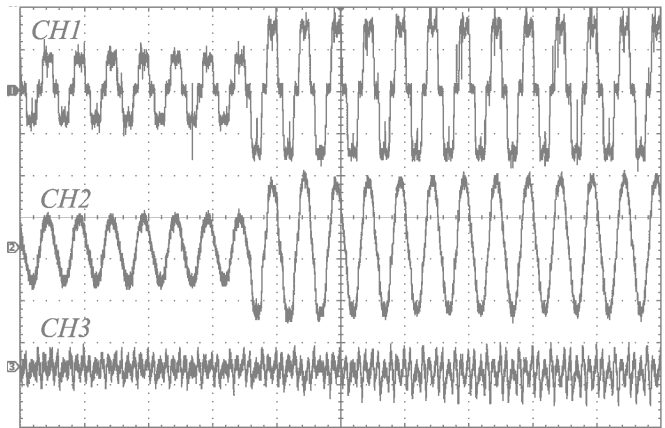

(a)

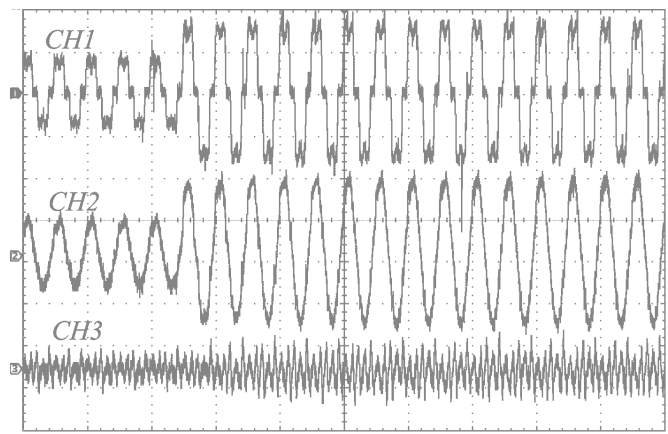

(b)

Fig. 21. Test results of transient responses in mode 2, with same load increase jump. Scale: $20 \mathrm{~A} / \mathrm{div}$ and $40 \mathrm{~ms} / \mathrm{div}$. CH1 is the load current; $\mathrm{CH} 2$ is the source current; $\mathrm{CH} 3$ is the compensating current. (a) Transient responses of scheme with VR controller. (b) Transient responses of scheme with PR controller.

Note that large errors appear for the 35th-order harmonic current in Fig. 19(b), whose reference magnitude is the same as the 25th order. That's because limited sampling would introduce non-negligible frequency deviation to the reference, causing great gain lost.

2) Mode 2

In this mode, three-phase VSI is controlled as selective APF. The nonlinear load is formed by a diode rectifier with resistor loads in the DC side (values are listed in Table II), injecting typical $6 k_{h} \pm 1$ order harmonic currents to the source. These harmonics are extracted from load current by the reference generator in Fig. 1, forming mixed current reference. Cascaded resonant controllers that up to the 19th order are implemented and the corresponding harmonics compensation results are subsequently presented.

When selectivity is not that important and all harmonics up to the 19th order need to be compensated, both PR and VR controllers exhibit similar excellent filtering results (see Fig. 20(a)). In this condition, all cascaded controllers up to the 19th order are kept for both resonant controllers, and larger proportional terms are selected $\left(k_{P R}=20, k_{V R}=6\right)$ to ensure good performance. The spectrums of load current, compensating current, and source current are obtained by power analyzer HIOKI 3197 (see Fig. 20(b)).

Due to selection of large proportional terms, good transient response speed is assured for both controllers. In the tests, the nonlinear load gave a $40 \%$ load increase jump. The transient responses of the VR and PR schemes are depicted in Figs. 21(a) and 21(b), respectively. Both schemes could track the change within one fundamental cycle, and good filtering performances are presented.

To check the selectivity, the resonant controller that tuned on fifth-order harmonic is removed from both cascaded resonant controllers. Considering that the mixed current reference is employed, the fifth-order component is still included in the reference. The test results are shown in Fig. 22 for the VR controller and Fig. 23 for the PR controller.

The scheme with VR controller exhibits good selectivity in Fig. 22(a), with $k_{P R}=6$ for each component. It outputs compensating current just without fifth-order. Therefore, all harmonics, except the fifth order, are well compensated in the source current. Corresponding spectrums of source current and compensating current are shown in Fig. 22(b). 


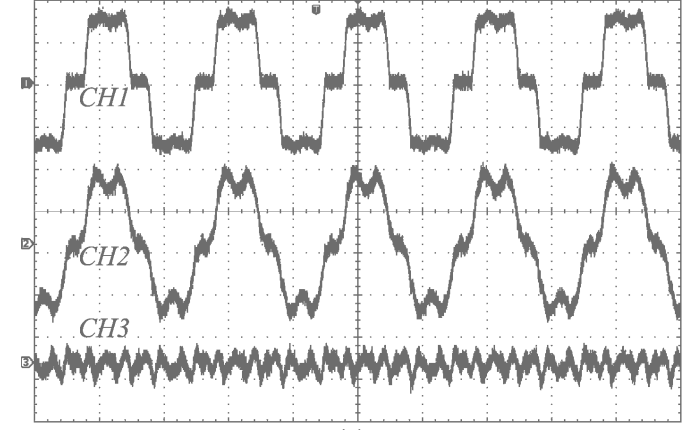

(a)

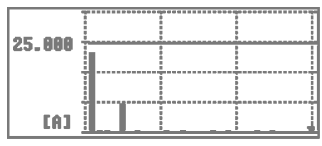

Spectrum

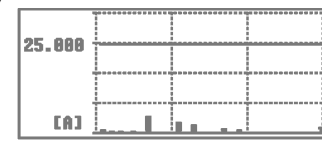

Spectrum 2

(b)

Fig. 22. Test results of the selectivity of VR controller in mode 2 , with the controller tuned on fifth-order component removed. (a) Waveforms with scale: $20 \mathrm{~A} / \mathrm{div}, 10 \mathrm{~ms} / \mathrm{div}$. $\mathrm{CH} 1$ is the load current; $\mathrm{CH} 2$ is the source current; $\mathrm{CH} 3$ is the compensating current. (b) Corresponding spectrums with scale of $500 \mathrm{~Hz} /$ div. Spectrum 1 represents the source current. Spectrum 2 represents the compensating current.

On the contrary, Fig. 23 shows the performance of scheme with PR controller, which has poorer selectivity. That's because current controller has degraded back to proportional controller for the fifth-order component. With $k_{P R}=20$, there is still large content of fifth-order harmonic current output by APF. Meanwhile, large steady-state errors appear (still large content of fifth-order harmonic remains in the source current), due to finite gain from proportional controller for this component. The selectivity could be hardly improved by reducing $k_{P R}$ because19th-order harmonic is selected, and further reducing of $k_{P R}$ will lead the system easily unstable in the test.

\section{CONCLUSIONS}

With the purpose of implementing for selective APF with mixed current reference, this paper presents specific comparison analysis between PR and VR controllers. Through comparisons, the following conclusions could be obtained:

1) The PR controller is equivalent to the SRF PI controller, whereas the VR controller is equivalent to the SRF VPI controller, which considers the cross-coupling influences in SRF.

2) The PR controller has poorer selectivity, and its performance is sensitive to the noises in the reference. By contrast, the VR controller provides good selectivity and robustness.

3) The selectivity of PR controller could be relatively improved by reducing its proportional term. However, this method has limitation from system stability: its proportional

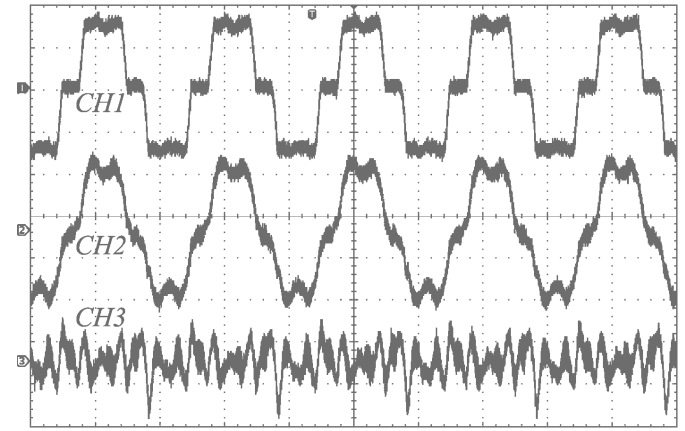

(a)

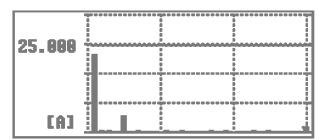

Spectrum 1

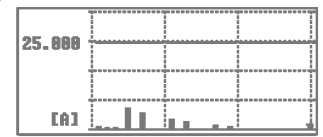

Spectrum 2 (b)

Fig. 23. Test results of the selectivity of PR controller in mode 2, with controller tuned on fifth-order component removed. (a) Waveforms with scale: $20 \mathrm{~A} / \mathrm{div}, 10 \mathrm{~ms} / \mathrm{div}$. $\mathrm{CH} 1$ is the load current; $\mathrm{CH} 2$ is the source current; $\mathrm{CH} 3$ is the compensating current. (b) Corresponding spectrums with scale of $500 \mathrm{~Hz} /$ div. Spectrum 1 represents the source current. Spectrum 2 represents the compensating current.

term has upper and lower limits from the view of stability, and the lower limit will increase with growth of the resonant frequency. Therefore, when higher order harmonic is selected, its selectivity could be hardly improved. On the contrary, the proportional term of VR controller has only an upper limit, which makes it possible to be flexibly adjusted with trade-off consideration of system stability, selectivity, and transient speed.

4) With VR controller, APF could compensate higher order harmonics than the scheme with PR controller.

Therefore, it could be concluded that, the PR controller should be implemented in the condition with accurate reference and lower need of selectivity, whereas the VR controller is much suitable for applications with strict demand of selectivity, such as selective APF with mixed current reference.

\section{APPENDIX A}

\section{SIMPLIFIED FREQUENCY DOMAIN ROTATION TRANSFORMATION}

To simplify the analysis, the simplified frequency domain rotation transformations between SRF and stationary frame are deduced here.

With the complex vector notion in [24], variables relation in stationary frame is defined as (A1) in frequency domain, where $X$ denotes related three-phase symmetrical current or voltage, and subscripts $\alpha$ and $\beta$ indicate the values of the two axes in stationary frame, respectively.

$$
X_{\alpha}(s)=j X_{\beta}(s)
$$


Taking positive sequence component as an example, rotation transformation from $\alpha \beta$ frame to SRF in the time domain could be expressed as (A2), where the subscripts $d$ and $q$ represent the variables in SRF that are rotating with angular frequency $\omega$.

$$
\left[\begin{array}{l}
x_{d}(t) \\
x_{q}(t)
\end{array}\right]=\left[\begin{array}{cc}
-\sin \omega t & \cos \omega t \\
\cos \omega t & \sin \omega t
\end{array}\right]\left[\begin{array}{l}
x_{\alpha}(t) \\
x_{\beta}(t)
\end{array}\right]
$$

By using the Euler formula to (A.2), we can express the rotations transformation as

$$
\begin{aligned}
& {\left[\begin{array}{l}
x_{d}(t) \\
x_{q}(t)
\end{array}\right]=\frac{1}{2}\left[\begin{array}{cc}
j\left(e^{j \omega t}-e^{-j \omega t}\right) & \left(e^{j \omega t}+e^{-j \omega t}\right) \\
\left(e^{j \omega t}+e^{-j \omega t}\right) & -j\left(e^{j \omega t}-e^{-j \omega t}\right)
\end{array}\right]\left[\begin{array}{l}
x_{\alpha}(t) \\
x_{\beta}(t)
\end{array}\right]} \\
& =\frac{1}{2}\left(e^{j \omega t}\left[\begin{array}{cc}
j & 1 \\
1 & -j
\end{array}\right]\left[\begin{array}{l}
x_{\alpha}(t) \\
x_{\beta}(t)
\end{array}\right]+e^{-j \omega t}\left[\begin{array}{cc}
-j & 1 \\
1 & j
\end{array}\right]\left[\begin{array}{l}
x_{\alpha}(t) \\
x_{\beta}(t)
\end{array}\right]\right) .
\end{aligned}
$$

The following rotation transformation in the frequency domain is obtained by applying the Laplace transformation to (A.3):

$$
\begin{aligned}
& {\left[\begin{array}{l}
X_{d}(s) \\
X_{q}(s)
\end{array}\right]=\frac{1}{2}\left(\left[\begin{array}{cc}
j & 1 \\
1 & -j
\end{array}\right]\left[\begin{array}{l}
X_{\alpha}(s-j \omega) \\
X_{\beta}(s-j \omega)
\end{array}\right]+\left[\begin{array}{cc}
-j & 1 \\
1 & j
\end{array}\right]\left[\begin{array}{l}
X_{\alpha}(s+j \omega) \\
X_{\beta}(s+j \omega)
\end{array}\right]\right)} \\
& =\frac{1}{2}\left[\begin{array}{c}
j X_{\alpha}(s-j \omega)+X_{\beta}(s-j \omega)-j X_{\alpha}(s+j \omega)+X_{\beta}(s+j \omega) \\
X_{\alpha}(s-j \omega)-j X_{\beta}(s-j \omega)+X_{\alpha}(s+j \omega)+j X_{\beta}(s+j \omega)
\end{array}\right] .
\end{aligned}
$$

Considering relation (A1), the rotation transformation becomes

$$
\left[\begin{array}{l}
X_{d}(s) \\
X_{q}(s)
\end{array}\right]=\left[\begin{array}{c}
X_{\beta}(s+j \omega) \\
X_{\alpha}(s+j \omega)
\end{array}\right] .
$$

Furthermore, the equation could be simplified as follows:

$$
X_{d q}(s)=T_{P}^{+}\left[X_{\alpha \beta}(s)\right]=X_{\alpha \beta}(s+j \omega),
$$

where $T$ represents rotation transformation in frequency domain; the subscript $P$ is defined for positive sequence components; $N$ is defined for negative sequence components; superscript + is defined for transformation from $\alpha \beta$ frame to $\mathrm{SRF},-$ for the inverse transformation.

In a similar manner, inverse transformation from SRF to $\alpha \beta$ frame for positive sequence component could be easily obtained. With transformation (A2), the symmetrical variables in the SRF have relation (A7) in the frequency domain.

$$
X_{q}(s)=j X_{d}(s)
$$

Therefore, the inverse rotation transformation in the frequency domain could be expressed as

$$
X_{\alpha \beta}(s)=T_{P}^{-}\left[X_{d q}(s)\right]=X_{d q}(s-j \omega) .
$$

For negative sequence components, both direction transformations could be expressed as (A9) and (A.10) by substituting $\omega$ as $-\omega$ in (A.6) and (A8), respectively.

$$
X_{d q}(s)=T_{N}^{+}\left[X_{\alpha \beta}(s)\right]=X_{\alpha \beta}(s-j \omega)
$$

$$
X_{\alpha \beta}(s)=T_{N}^{-}\left[X_{d q}(s)\right]=X_{d q}(s+j \omega)
$$

The expressions from (A6) and (A8) to (A10) denote that rotation transformation is a kind of frequency shift process in the frequency domain.

\section{REFERENCES}

[1] P. Mattavelli, "A closed-Loop selective harmonic compensation for active filters," IEEE Trans. Ind. Appl., Vol. 37, No. 1, pp. 81-89, Jan./Feb. 2001.

[2] D. Yazdani, A. Bakhshai, G. Joós, M. Mojiri, "A real-time three-phase selective harmonic-extraction approach for grid-connected converters," IEEE Trans. Ind. Electron., Vol.56, No.10, pp. 4097-4106. Oct. 2009.

[3] Reyes S. H., Patricio S., Hyosung K., "Instantaneous reactive power theory applied to active power filter compensation: different approaches, assessment, and experimental results," IEEE Trans. Ind. Electron., Vol. 55, No. 1, pp. 184-196, Jan. 2008.

[4] Hongyu Li, Fang Zhuo, Zhaoan Wang, Wanjun Lei, Longhui $\mathrm{Wu}$, "A novel time-domain current-detection algorithm for shunt active poer filters," IEEE Trans. Power System, Vol. 20, No.2, pp. 664-651, May 2005.

[5] C. Lascu, L. Asiminoaei, I. Boldea, and F. Blaabjerg, "High performance current controller for selective harmonic compensation in active power filters," IEEE Trans. power Electron., Vol. 27, No. 5, pp. 1826-1835, Sep. 2007.

[6] M. J. Newman, D. N. Zmood and D. G. Holmes, "Stationary frame harmonic reference generation for active filter systems," IEEE Trans. Ind. Appl., Vol. 38, No. 6, pp.1591-1599, Nov./Dec. 2002.

[7] R. I. Bojoi, G. Griva, M. Guerriero, F. Farina, and F. Profumo, "Current control strategy for power conditioners using sinusoidal signal integrators in synchronous reference frame," IEEE Trans. Power Electron., Vol. 20, No. 6, pp. 1402-1412, Nov. 2005.

[8] A. Chandra, B. Singh, B. N. Singh, K. Al-Haddad, "An improved control algorithm of shunt active filter for vltage regulation harmonic elimination, power-factor correction, and balancing of nonlinear loads," IEEE Trans. Power Electron., Vol. 15, No. 3, pp. 495-507, May 2000.

[9] S. Fukuda, R. Imamura, "Application of sinusoidal internal model to current control of three-phase utility-interface converters," IEEE Trans. Ind. Electron., Vol. 52, No. 22, pp. 420-426, Apr. 2005.

[10] C. D. Schauder and R. Caddy, "Current control of volgage-source inverters for fast four-quadrant drive performance," IEEE Trans. Ind. Appl., Vol. IA-18, pp. 163-171, Mar./Apr. 1982

[11] T. R. Rowan and R. L. Kerman, "A new synchronous current regulator and an analysis of current-regulated PWM inverters," IEEE Trans. Ind. Appl., Vol. IA-22, pp. 678-690, July/Aug. 1986

[12] D. N. Zmood, and D. G. Holmes, "Stationary frame current regulation of PWM inverters with zero steady-state error," IEEE Trans. Power Electron., Vol. 18, No. 3, pp. 814-822, May 2003.

[13] X. Yuan, W. Merk, H. Stemmler, and J. Allmeling, "Stationary-frame generalized integrators for current control of active power filters with zero steady-state error for current harmonics of concern under unbalanced and 
distorted operating conditions," IEEE Trans. Ind. Appl., Vol. 38, No. 2, pp. 523-532, Mar./Apr. 2002.

[14] C. Lascu, L. Asiminoaei, I. Boldea, and F. Blaabjerg, "Frequency response analysis of current controllers for selective harmonic compensation in active power filters," IEEE Trans. Ind. Electron., Vol. 56. No. 2, pp. 337-347, Feb. 2009.

[15] A. G. Yepes, F. D. Freijedo, O. Lopez, and J. Doval-Gandoy, "High-performance digital resonant controllers implemented with two integrators," IEEE Trans. Power Electron., Vol. 26, No. 2, pp. 563-576, Feb. 2011.

[16] A. G. Yepes, F. D. Freijedo, O. Lopez, and J. Doval-Gandoy, "Analysis and design of resonant current controllers for voltage-source converters by means of nyquist diagrams and sensitivity function," IEEE Trans. Ind. Electron., Vol. 58, No. 11, pp. 5231-5250, Nov. 2011.

[17] S. Fukuda and T. Yoda, "A novel current-tracking method for active filters based on sinusoidal internal model," IEEE Trans. Ind. Appl., Vol. 37, No. 3, pp. 888-895, May/Jun. 2001.

[18] M. Castilla, J. Miret, J. Matas, L. G. de Vicuna, and J. M. Guerrero, "Linear current control scheme with series resonant harmonic commpensator for single-phase grid-connected photovoltaic inverters," IEEE Trans. Ind. Electron., Vol. 45, No. 7, pp.2724-2733, Jul. 2008.

[19] R. Bojoi, L. Limongi, F. Profumo, D. Roiu, and A. Tenconi, "Analysis of current controllers for active power filters using selective harmonic compensation schemes," IEEJ Trans. Electr. Electron. Eng., Vol. 4, No.2, pp.139-157, 2009.

[20] L. Limongi, R. Bojoi, G. Griva, and A. Tenconi, "Digital current-control schemes," IEEE Trans. Ind. Electron. Mag., Vol. 3, No. 1, pp. 20-31, Mar. 2009.

[21] M. Liserre, R. Teodorescu, and F. Blaabjerg, "Multiple harmonics control for three-phase grid converter systems with the use of PI-RES current controller in a rotating frame," IEEE Trans. Power Electron., Vol. 21, No. 3, pp. 836-841, May 2006

[22] M. Castilla, J. Miret, J. Matas, L. G. de Vicuna and J. M. Guerrero, "Control design guidelines for single-phase grid connected photovoltaic inverters with damped resonant harmonic compensators," IEEE Trans. Ind. Electron., Vol. 56, No. 11, pp. 4492-4501, Nov. 2009

[23] W. Lenwari, M. Sumner, P. Zanchetta, "The use of genetic algorithms for the design of resonant compensators for active filters," IEEE Trans. Ind. Electron., Vol. 56, No. 8, pp. 2852-2861, Aug. 2009

[24] F. Briz, M. W. Degner, and R. D. Lorenz, "Analysis and design of current regulators using complex vectors," IEEE Trans. Ind. Appl., Vol.36, No. 3, pp. 817-825, May/Jun. 2000.

[25] G. F. Franklin, J. D. Powell, and A. Emami-Naeini, Feedback Control of Dynamic System. Kelhi, India: Pearson Educ., 2002.

[26] G. C. Goodwin, S. F. Graebe, and M. E. Salgado, Control System Design. Englewood Cliffs, NJ: Prentice-Hall, 2000.

[27] A. G. Yepes, F. D. Freijedo, J.Doval-Gandoy, O. Lopez, J. Malvar, and P. Fernandez-Comesaana, "Effects of discretization methods on the performance of resonant controllers," IEEE Trans. Power Electron., Vol. 25, No. 7, pp. 1692-1712, Jul. 2010

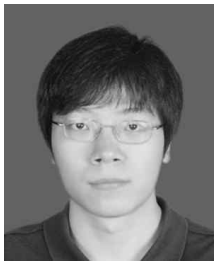

Hao Yi was born in Shaanxi, China, in 1984. $\mathrm{He}$ received his BS degree in Electrical Engineering from the Hebei University of Technology, Tianjin, China, in 2007 and his MS degree in Electrical Engineering from Xi'an Jiaotong University, Xi'an, China. He is currently pursuing his $\mathrm{PhD}$ degree in Electrical Engineering at the Faculty of Industry Automation, School of Electrical Engineering, Xi'an Jiaotong University. His research interests include modeling and control of high-power converter, power quality improvement, and control of multilevel converter. He is currently working on improving the performance of active power filters.

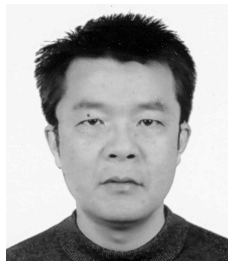

Fang Zhuo was born in Shanghai, China, on May, 1962. He received his BS degree in Automatic Control from Xi'an Jiaotong University in 1984, and then he joined Xi' an Jiaotong University. In 1989 and 2001, he received his $\mathrm{MS}$ and $\mathrm{PhD}$ degrees, respectively, in Automation and Electrical Engineering in Xi' an Jiaotong University. In 2004, he worked as a visiting scholar in Nanyang Technological University. He was an associate professor in Xi' an Jiaotong University in 1996 and a full-time professor in Power Electronics and Drives in 2004. He was then employed as a supervisor of $\mathrm{PhD}$ students. He is also the associate dean of the Faculty of Industry Automation. Dr. Zhuo's research interests include power electronics, power quality, active power filter, reactive power compensation, and inverters for distributed and power generation. He is the author or coauthor of more than 160 publications, more than 30 papers was indexed by SCI, EI, and ISTP, in his research fields. He is also the coauthor of two handbooks. He is the key finisher of four projects sponsored by the National Natural Science Foundation of China, and more than 40 projects cooperated with companies from the industry. He is the owner of four provincialand ministerial-level science and technology advancement awards and four patents. Professor Zhuo is a member of IEEE, China Electro-technical Society, Automation Society, and Power Supply Society. In addition, he is the power quality professional chairman of Power Supply Society in China.

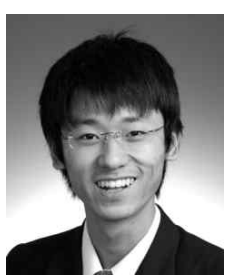

Yu Li was born in Shaanxi, China, in 1987. $\mathrm{He}$ received his $\mathrm{BS}$ and $\mathrm{MS}$ degrees in Electrical Engineering from Xi'an Jiaotong University, Xi'an, China, in 2010 and 2013, respectively. $\mathrm{He}$ is currently an Electrical Engineer of Bosch Rexroth (Xi'an) Electric Drives and Controls Co., Ltd. His research interests include the design, control, and application of Active Power Filter, including harmonic current detection and three-level APF control. 


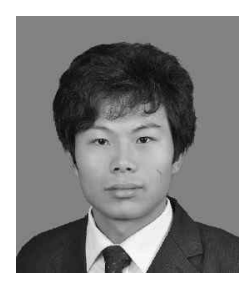

Yanjun Zhang was born in Henan, China, in 1988. He received his BS degree from Huazhong University of Science and Technology, Wuhan, China, in 2010. He is currently pursuing his MS degree in Electrical Engineering at Xian Jiaotong University, Xi'an, China. His current research includes power quality, active power filters, and static VAR generators.

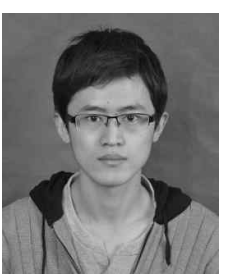

Wenda Zhan was born in Zhejiang, China, in 1989. He received his BS degree in Electrical Engineering from Xi'an Jiaotong University, Xi'an, China, in 2011, and is currently pursuing his MS degree at Power Electronics and Renewable Energy Research Center (PEREC), School of Electrical Engineering, Xi'an Jiaotong University, $\mathrm{Xi}$ 'an, China. He is currently a research assistant. His research interests include the design, control, and applications of active power filters, multilevel converters, and repetitive control. 